\title{
On Stabilization of PVPA/PVA Electrospun Nanofiber Membrane and Its Effect on Material Properties and Biocompatibility
}

\author{
Rose Ann Franco, Young-Ki Min, Hun-Mo Yang, and Byong-Taek Lee \\ Department of Biomedical Engineering and Materials, Soonchunhyang University, Cheonan-si 330-090, Republic of Korea \\ Correspondence should be addressed to Byong-Taek Lee, lbt@sch.ac.kr
}

Received 25 November 2011; Accepted 9 December 2011

Academic Editor: Chunyi Zhi

Copyright (๑) 2012 Rose Ann Franco et al. This is an open access article distributed under the Creative Commons Attribution License, which permits unrestricted use, distribution, and reproduction in any medium, provided the original work is properly cited.

A novel nanofiber membrane was fabricated by electrospinning composed of polyvinyl phosphonic acid (PVPA) and polyvinyl alcohol (PVA). Stabilization was done due to the high dissolvability of the membrane when in contact with water. Physical treatment was done by exposure to heat at $150^{\circ} \mathrm{C}$ in a vacuum environment at different periods of time. Chemical crosslinking was done by immersion in methanol and methanol/ glutaraldehyde. A heat-exposed membrane was also further crosslinked chemically. All conditions were compared with regards to its effect on the material properties of the membranes and its biological response in vitro with MG-63 osteoblast-like cell line. Visual examination and dimensional analyses showed that heat treatment produced discoloration on the membrane surface and chemical crosslinking reduced membrane dimensions. Tensile strength and strain improved in crosslinked membranes compared to noncrosslinked counterpart. Swelling and degradation was also investigated and was seen to vary depending on the crosslinking condition. Biocompatibility was observed to be more favorable in heat-treated membranes.

\section{Introduction}

Electrospun membranes comprise a major part of scaffolds being studied for tissue engineering [1] due to its resemblance to the native extracellular matrix at the microscopic level [2]. The method of electrospinning is clearly advantageous since it can be manipulated in a variety of ways depending on the desired application. Adjustment of solution concentration, injection rate, supplied voltage, and collecting distance was shown to have an effect on the resulting fiber morphology [3]. The mechanical property, hydrophilicity and hydrophobicity and degradation of the membranes depend on the starting material used [4]. A wide range of polymers, both of natural and of synthetic origin, have been utilized in electrospinning for different target organ applications. In this study, an electrospun membrane using a novel polymer combination was fabricated with an aim to be used in tissue engineering applications.

Polyvinylphosphonic acid (PVPA) is an acidic polymer that shares a similar structural formula to poly(alkenoic acids) [5]. It is synthesized by free-radical polymerization of vinylphosphonic acid (VPA) or initial polymerization of VPA followed by hydrolysis of the polymer [6,7]. It is used as an additive hardening ingredient in dental cements which has a quality similar to that of glass polyalkenoates [8]. Aside from its hardening properties, the abundance of phosphonate groups of PVPA has been shown to be beneficial due to its similarity to the phosphate groups of the natural bone hydroxyapatite [9], which can assist in the bone-bonding between the restorative material and the native bone tissue.

Polyvinyl alcohol (PVA) is a polymer widely used in the medical and food industry. PVA has been used solely and combined as a starting material in electrospinning for different applications, such as tissue engineering scaffolds and drug delivery systems [10-14]. This polymer has been shown to possess mechanical reliability and the ability to support cell adhesion and growth. In this study, PVA was combined with PVPA to fabricate a novel electrospun nanofiber membrane. However, the initial characterization of the fabricated membrane showed compromised strength and high water dissolvability. In this instance, crosslinking and stabilization of the membrane was a necessity. Two methods were 
used: physical stabilization by heat treatment at $150^{\circ} \mathrm{C}$ in a vacuum environment and chemical crosslinking by immersion in methanol and combined methanol/glutaraldehyde. The effects of the crosslinking methods with regards to the material property and biocompatibility were examined.

\section{Materials and Methods}

2.1. Reagents. Polyvinyl phosphonic acid (PVPA, 30\% solution) was obtained from Polysciences, Inc. Poly(vinyl alcohol, fully hydrolyzed) (PVA) was purchased from SigmaAldrich., methanol from Merck, Co., glutaraldehyde, 25\% from Shiwa, Korea., and dimethysulfoxide (DMSO, 99.0\%) from Samchun Pure Chemical Co., LTD, Republic of Korea. Dulbecco's Modified Eagle Medium (DMEM) was purchased from HyClone Thermo Scientific. Fetal bovine serum (FBS), Penicillin and Streptomycin antibiotics, Dulbecco's phosphate buffered saline (PBS, without calcium and magnesium), MTT solution, and Trypsin-EDTA were purchased from GIBCO (Carlsbad, CA). Hexamethyldisilazane (HMDS) was from Sigma, USA. The water used in the entire experiment was deionized through Milli-Q System (Millipore, USA). All other chemicals used were of reagent grade.

2.2. Membrane Fabrication. PVPA was diluted to $0.1 \%$ with addition of deionized water and heated to $80^{\circ} \mathrm{C}$. PVA, $12 \% \mathrm{w} / \mathrm{v}$, was added and dissolved at $80^{\circ} \mathrm{C}$ until no solid particles remained. The solution was mechanically stirred and boiled until completely homogenous. The solution was electrospun with solution flow rate of $0.5 \mathrm{~mL} / \mathrm{hr}$, voltage application of $20 \mathrm{kV}, 12 \mathrm{~cm}$ distance from 19-G needle to metal collector, and $150 \mathrm{rpm}$ speed of the collecting drum wrapped with aluminum foil. The thickness was optimized at around $1.2 \mathrm{~mm}$ with a fixed amount of solution.

2.3. Membrane Stabilization and Crosslinking. Membrane stabilization was done by heat treatment and chemical crosslinking by methanol and combination of methanol/glutaraldehyde to compare the optimum condition in strengthening the membranes. Heat stabilization was done by placing the membrane in a vacuum furnace oven set at $150^{\circ} \mathrm{C}$ for 2 , 10 and 24 hours (P2H, P10H, and $\mathrm{P} 24 \mathrm{H}$, resp.). Chemical crosslinking was done by immersing the membrane in absolute methanol and methanol with $1 \%$ glutaraldehyde. A membrane that was heat-stabilized for $24 \mathrm{~h}$ was subsequently immersed in methanol to determine the effect of combined treatment methods.

\subsection{Material Characterization}

2.4.1. Morphological and Dimensional Analyses. Morphological changes in the membranes were noted before and after treatment by optical inspection and microscopic visualization. Properties such as water dissolvability, discoloration, and size reduction were observed.

Nanofiber membranes were sputtered with platinum sputter coater (Cressington Sputter Coater) and were viewed using field emission scanning electron microscope
(JSM701F, JEOL, Japan) with acceleration voltage of $15 \mathrm{kV}$. The fiber diameters were measured with an accompanying image analyzer. The average fiber diameters were determined from a random sample lot $(n=50)$ across different sites of the fabricated membrane.

Calculations on the change in surface area, thickness, and density were calculated following measurements made on the membrane dimensions before and after the crosslinking methods were applied. The surface area and thickness were measured with four replicates. About 50 fibers $(n=50)$ were measured across different membrane sites.

2.4.2. Mechanical Strength. Assessment of the mechanical property of the PVPA/PVA and PVA nanofiber membranes was done by measuring the tensile strength with a universal testing machine (R\&B Co., Ltd., Republic of Korea). An accompanying Helio-X software program determined the tensile strength and strain values of the materials. Rectangular strips of $33 \mathrm{~mm} \times 2 \mathrm{~mm} \times T$ (where $T$ is the thickness) were cut from each sample of electrospun PVPA/PVA and PVA membranes. Thickness of the membrane was determined by photomicrographs from SEM and scaled to measure. The ends of membrane strips were glued to a paper frame to anchor it to the tensile tester. The sides of the frame were cut upon application of strain at crosshead speed of $1 \mathrm{~mm} / \mathrm{min}$ with $1 \mathrm{~kg}$ load cell.

2.4.3. Swelling Property. Preweighed, treated PVPA/PVA membranes were cut in circles with $1 \mathrm{~mm}$ diameter and were placed in a 24-well tissue culture plate containing $1 \mathrm{~mL}$ of PBS ( $\mathrm{pH} 7.4$ ) per well. PBS-immersed membranes were incubated at $37^{\circ} \mathrm{C}$ for 1 hour and 24 hours. Membranes were immediately blotted with filter paper to absorb excess water and then weighed. Immersion in PBS was extended for 14 days to determine weight changes.

\subsection{Biocompatibility}

2.5.1. Cell Culture. MG-63 osteoblast-like cells were obtained from Korean Cell Line Bank and were subcultured per laboratory procedures [15]. Cells were washed with PBS, dissociated with trypsin-EDTA, and then centrifuged. Supernatant was discarded and cells were resuspended with DMEM with $10 \%(\mathrm{v} / \mathrm{v})$ of fetal bovine serum (FBS) and $1 \%$ penicillin/streptomycin antibiotics in tissue culture flasks (Nunc) at $37^{\circ} \mathrm{C}, 5 \% \mathrm{CO}_{2}$, and $95 \%$ humidity. Culture medium was replaced every two days.

2.5.2. Cytotoxicity. Determination of the material cytotoxicity was done following the ISO 10993-5 protocol. Membranes, $20 \mathrm{~mm} \times 40 \mathrm{~mm}$, were incubated in DMEM media to obtain extract solutions after 1 day of incubation with shaking at $37^{\circ} \mathrm{C}$ humidified environment. Extract solutions were filtered with $0.20 \mu \mathrm{m}$ DISMIC filter (Advantec) for sterilization and then diluted with fresh media to obtain different concentrations: $12.5 \%, 25 \%, 50 \%$, and 100\%. A fresh media without extract solution was designated as control $(0 \%)$. Diluted and nondiluted extract solutions 
TABLE 1: Crosslinking conditions and morphological evaluation of the membranes.

\begin{tabular}{lccccc}
\hline Sample & Crosslinking condition & Exposure time & Dissolvability in water & Discoloration & Size reduction \\
\hline P0 & $\mathrm{x}$ & $\mathrm{x}$ & $(+)$ & $\mathrm{x}$ & $\mathrm{x}$ \\
PH2 & Heat $\left(150^{\circ} \mathrm{C}\right)$ & 2 hours & $(-)$ & $(+)$ & $(-)$ \\
PH10 & Heat $\left(150^{\circ} \mathrm{C}\right)$ & 10 hours & $(-)$ & $(++)$ & $(-)$ \\
PH24 & Heat $\left(150^{\circ} \mathrm{C}\right)$ & 24 hours & $(-)$ & $(+++)$ & $(-)$ \\
PM & Methanol & 15 mins & $(-)$ & $(-)$ & $(++)$ \\
PMG & Methanol/glutaraldehyde & 15 mins & $(-)$ & $(-)$ & $(++)$ \\
PH24M & Heat $\left(150^{\circ} \mathrm{C}\right)+$ Methanol/glutaraldehyde & 24 hours +15 mins & $(-)$ & $(-)$ & $(+)$ \\
\hline
\end{tabular}

were then used to treat the MG63 osteoblast-like cells, $10^{4}$ cells $/ \mathrm{mL}$, grown for 24 hours. Optical density was quantified after another 24 hours using MTT assay. Cell viability was quantified as optical density of the treated wells relative to the nontreated wells $(0 \%)$.

2.5.3. Cell Proliferation Analysis. MG63 osteoblast-like cells, $10^{4}$ cells $/ \mathrm{mL}$, were seeded on the electrospun PVPA/PVA membranes that were $15 \mathrm{~mm}$ in diameter and were incubated for 1,3 , and 5 days in 24-well culture plates. Media were replaced every other day. After each incubation time, media were discarded and MTT solution was added as substrate and reincubated for another 4 hours of mitochondrial enzyme conversion. DMSO was then added to dissolve the formed formazan salts and plates were shaken for 1 hour. The absorbance was read using an ELISA plate reader (Tecan Infinite F50, Switzerland). Cell viability was equated with the obtained optical density at $595 \mathrm{~nm}$.

2.5.4. Cell Adhesion Behavior. SEM visualization of cell adhesion behavior was done by seeding electrospun $\mathrm{P} 24 \mathrm{H}$ membranes with MG63 osteoblast-like cells, $10^{4}$ cells $/ \mathrm{mL}$. Samples were incubated for 1 and 5 days. Cell-seeded samples were visualized under FE-SEM after glutaraldehyde fixation, dehydration with graded ethanol series and critical-point drying with hexamethyldisilazane.

Cell-seeded scaffolds were also viewed under laser scanning confocal microscope (Olympus FV 1000, Japan) after staining with DAPI (4'-6-Diamidino-2-phenylindole, Sigma) and DiOC $_{6}$ (3,3'-dihexyloxacarbocyanine iodide, Invitrogen).

2.6. Statistical Analyses. All experiments are done in replicates of 4 unless otherwise stated. Values are expressed as mean average of 4 replicates and standard deviation. Analysis of variance (ANOVA) was done to determine if differences in values were statistically significant.

\section{Results}

3.1. Morphological and Dimensional Analyses. Treatment of the PVPA/PVA membranes by both physical and chemical means showed morphological changes in the macroscopic appearance and the microstructure of the membrane. Table 1 enumerates the qualitative data obtained from visual observation on the membranes before and after crosslinking. Noncrosslinked PVPA/PVA membrane (P0) was highly dissolvable to water but this was prevented after crosslinking was done. Discoloration was observed in the membranes which underwent heat stabilization. The intensity of the discoloration was observed to increase when the exposure time was lengthened $(\mathrm{P} 2 \mathrm{H}<\mathrm{P} 10 \mathrm{H}<\mathrm{P} 24 \mathrm{H})$ (Figure 1). No discoloration was noted in the chemically crosslinked membranes. However, a notable reduction in the size was seen after 15 minutes immersion in methanol (PM) and methanol/glutaraldehyde (PMG) which reduced the membrane surface area to less than 50\% (Figure 1(b)). Membrane density was also seen to change with noted decrease in samples treated with heat but increased to about twofold when chemically treated (Figure 1(c)).

The microstructure of the membrane fibers was also observed after treatment and the changes were noted to vary depending on the method applied. The average fiber diameter of nontreated PVPA/PVA membrane was measured at $124.25 \pm 13.37 \mathrm{~nm}$. Figure $1(\mathrm{~d})$ shows the enlargement of membrane fibers after physical stabilization with relatively slight increase as the heat exposure was lengthened. Methanol and methanol/glutaraldehyde crosslinking increased membrane fibers by $40.20 \%$ and $30.35 \%$, respectively.

Figure 2 shows the SEM micrographs of representative samples after crosslinking. In comparison with the noncrosslinked fibers (Figure 2(a)), the heat-stabilized fibers appeared relatively flattened and more compressed compared to the nontreated sample (Figure 2(b)). Chemical crosslinking by methanol immersion produced a more compressed fibrous network which appeared swollen or melted (Figure 2(c)). It was noted that in P24HM (Figure 2(d)) the membranes appeared to retain its morphological integrity but fibers were relatively larger in appearance compared to noncrosslinked fibers. Membranes treated with similar conditions to the ones presented were no longer included due to similarity in morphology.

3.2. Mechanical Strength. Improvement of mechanical properties was observed to be varied for treated membranes when compared to the nontreated sample (Figure 3). It was observed that in the heat-stabilized membranes, stress improvement was not statistically significant $(P>0.05)$ for $\mathrm{P} 2 \mathrm{H}, \mathrm{P} 10 \mathrm{H}$, and $\mathrm{P} 24 \mathrm{H}$. However, strain values increased 


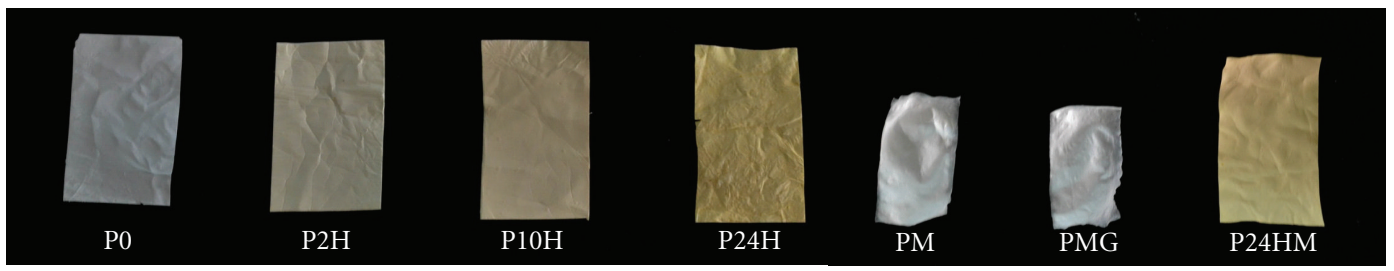

(a)

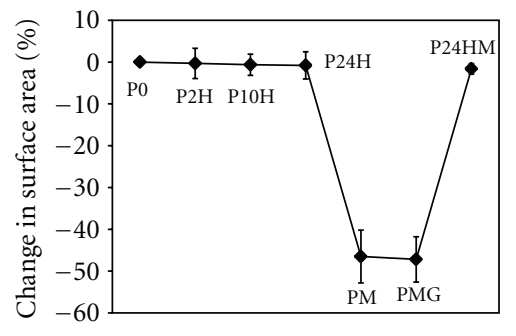

(b)

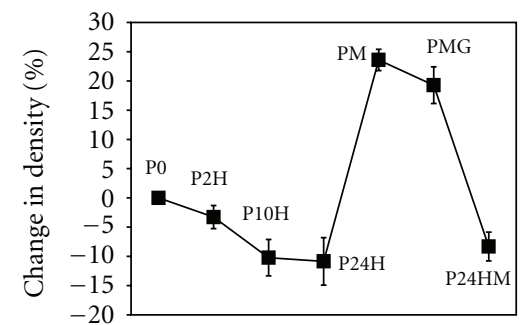

(c)

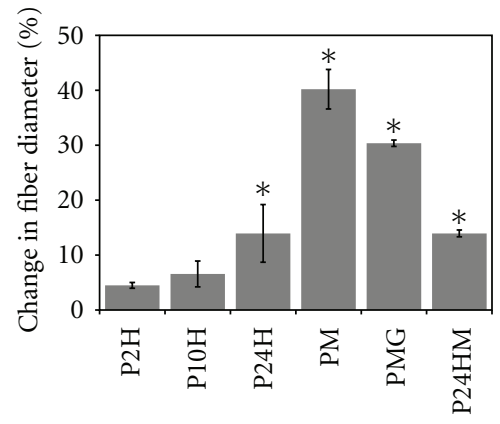

(d)

Figure 1: Optical image of non-crosslinked PVPA/PVA membrane (P0) with heat-stabilized samples (P2H, P10H, P24H), chemically crosslinked samples (PM, PMG), and membrane with combined crosslinking methods (P24HM) and its corresponding change in surface area (b), density (c), and fiber diameter (d) relative to the non-crosslinked membrane.

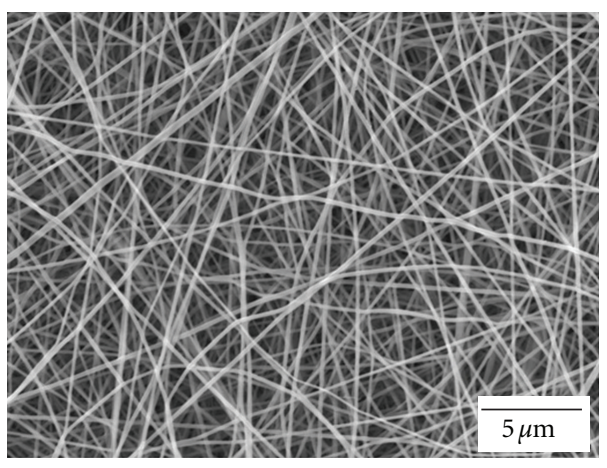

(a)

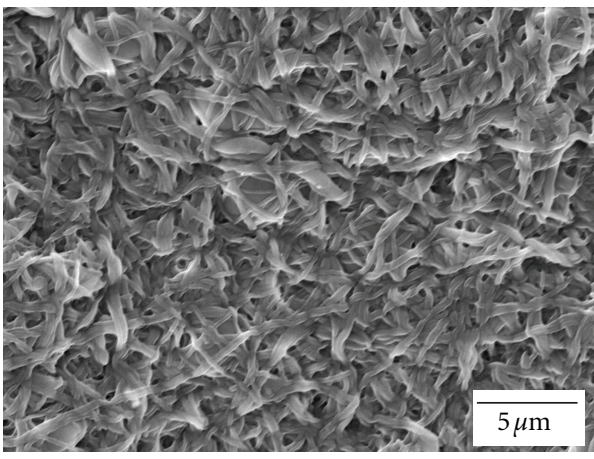

(c)

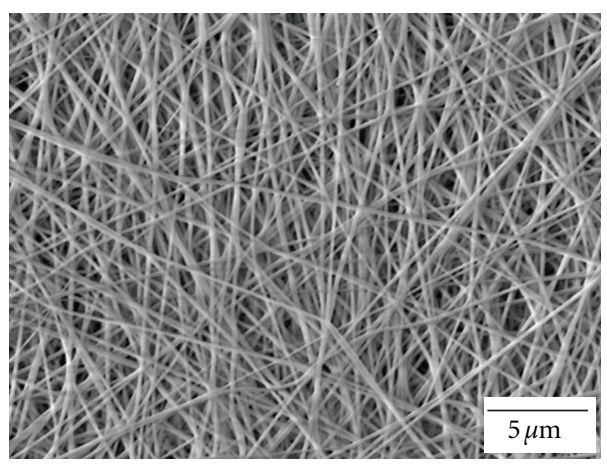

(b)

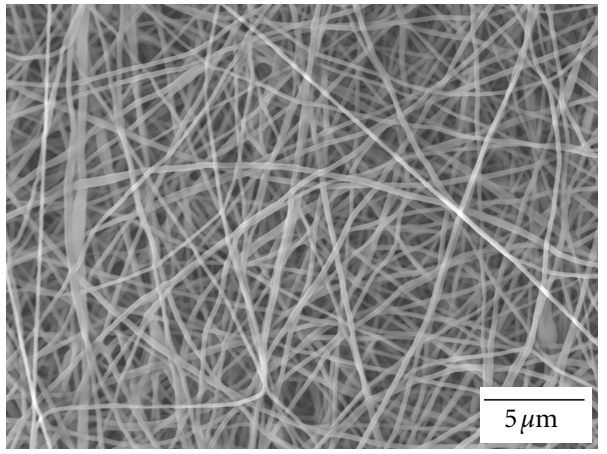

(d)

Figure 2: SEM micrographs of P0, noncrosslinked PVPA/PVA membrane (a), P24H, heat-stabilized for 24 hours (b), PM, methanolcrosslinked membrane (c), and P24HM, heat- and methanol-crosslinked membrane (d). 


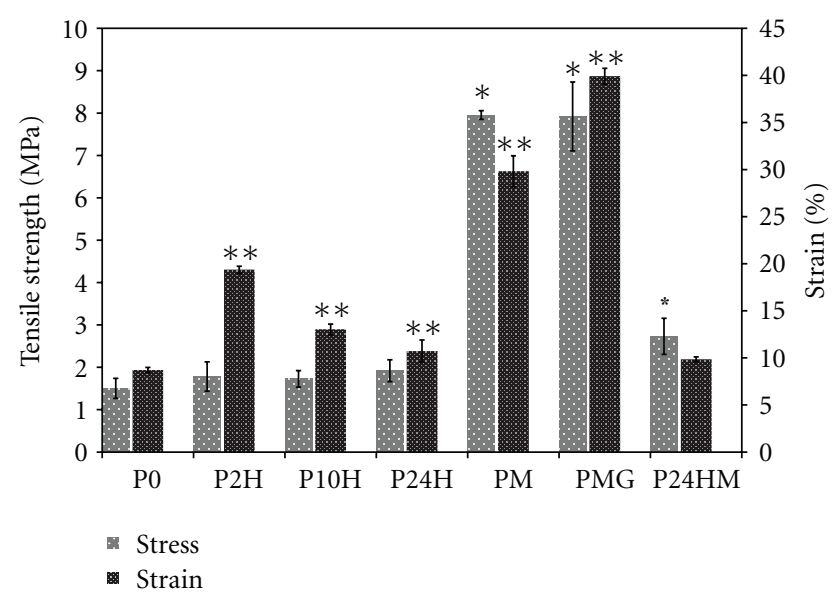

Figure 3: Stress and strain values of heat-stabilized samples (P2H, P10H, P24H), and methanol (PM), methanol/glutaraldehyde (PMG), and heat and methanol-crosslinked (P24HM) PVPA/PVA membranes compared to noncrosslinked membrane (P0). Statistically significant values are indicated for stress $\left({ }^{*}\right)$ and strain $\left({ }^{*}\right)$ as compared with $\mathrm{P} 0$ values.

upon crosslinking with higher values when crosslinking time was relatively shorter $(\mathrm{P} 2 \mathrm{H} 19.36 \%>\mathrm{P} 10 \mathrm{H} 13.03 \%>\mathrm{P} 24 \mathrm{H}$ $10.73 \%>$ P0 8.71\%).

Tensile strength values of methanol- (PM) and methanol/ glutaraldehyde- (PMG) crosslinked samples were significantly higher than the P0, but the difference between PM and PMG was not statistically significant $(P>0.05)(\mathrm{PM}=$ PMG > P0). Strain at break was observed to be more pronounced when glutaraldehyde was added to the methanol as seen in the increased strain value of PMG compared to PM. When PVPA/PVA membrane was initially heated and then treated with methanol for crosslinking (P24HM), stress improved compared to the heat-treated and noncrosslinked membranes but lower than the methanol-immersed membranes (PM, PMG > P24HM > P2H, P10H, P24H). The strain value of $\mathrm{P} 24 \mathrm{HM}$ was compared with the strain value of P0 using statistical analysis and the difference was not statistically significant $(P>0.05)$.

3.3. Swelling Property. Figure 4(a) shows the swelling capability of the treated PVPA/PVA membranes after 1-hour and 24-hour immersion in PBS. It was observed that the all membranes exhibited the capability to hold/attract water molecules. The methanol-immersed membranes displayed higher swelling ratios than its heat-stabilized counterparts. Swelling ratio for PM and PMG was more than 500\% and $600 \%$ after 1 hour and 24 hours, respectively. Heat-stabilized membranes showed a slight decrease in the swelling ratio as the length of exposure was increased. P24HM displayed swelling ratio higher than $\mathrm{P} 24 \mathrm{H}$ but significantly reduced compared to PM and PMG.

Immersion in the PBS was done for an extended period of 14 days to determine if there are weight changes. After sufficient drying, no significant weight change was noted but SEM micrograph showed swollen fiber network of $\mathrm{P} 24 \mathrm{H}$
(Figure 4(b1)) and flattened, film-like appearance of PM membrane (Figure 4(b2)). Membranes treated with similar conditions to the ones presented were no longer included due to similarity in morphology.

3.4. Material Cytotoxicity. Cytotoxicity of the membrane was determined by exposing cultured cells to diluted extract solutions of the cross-linked membranes as prescribed in ISO-10993-5 protocol. Cell response to the diluted extract solutions was shown in Figure 5. Cell viability was more than $80 \%$ at $100 \%$ extract solution for all heat-stabilized samples. However, significant reduction in cell viability was observed in samples treated with methanol and methanol/glutaraldehyde. Cell viability in wells treated with PM extract solutions was about $80 \%$ with $100 \%$ extract solution while it was about $60 \%$ with $100 \%$ extract solution of PMG. In comparison, cell viability increased in wells treated with extract solutions of P24HM with about $70 \%$ cell viability with $100 \%$ extract solution.

3.5. Cell Proliferation Analysis. Cell proliferation behavior of MG63 osteoblast-like cells was seen in Figure 6 wherein it was observed that varying the time of the heat exposure did not affect the cell growth as it showed values which are not statistically significant in all sampling days $\left({ }^{*} P>0.05\right)$. However, it was seen that membranes that were chemically crosslinked showed reduced biocompatibility even after several days of incubation. Particularly, PMG showed significant reduction of cell viability compared to other membranes after 1 day of incubation with an observed further decrease in the optical density after 3 days and 5 days of incubation.

3.6. Cell Adhesion Behavior. After characterization of the material properties and the biocompatibility of the treated membranes, $\mathrm{P} 24 \mathrm{H}$ membrane was chosen as an optimized condition and cell adhesion behavior was demonstrated using this membrane. Cell elongation and shape changes were observed after 1 day of incubation in $\mathrm{P} 24 \mathrm{H}$ membrane (Figures 7(a1) and 7(b1)). This growth was observed to be more pronounced after 5 days of culture with the extracellular material almost covering the majority of the membrane surface (Figures 7(a2) and 7(b2). Confocal images show increased cell density with the DAPI-stained nucleus from 1 day to 5 days of incubation (Figures $7(\mathrm{c} 1)$ and $7(\mathrm{c} 2)$ ).

\section{Discussion}

The novel combination of PVPA and PVA as an electrospun membrane showed promising characteristics as tissue engineering scaffold. PVA has already been used in different studies as a tissue engineering scaffold due to its favorable material properties [16]. However, PVA alone displays limited biocompatibility since cell adhesion to this material is compromised due to a lack of cell adhesion sites. Combinations with different polymers and surface modification have been used to improve the material properties and enable successful cell growth in these scaffolds [1720]. In this study, PVPA was combined with PVA due to 


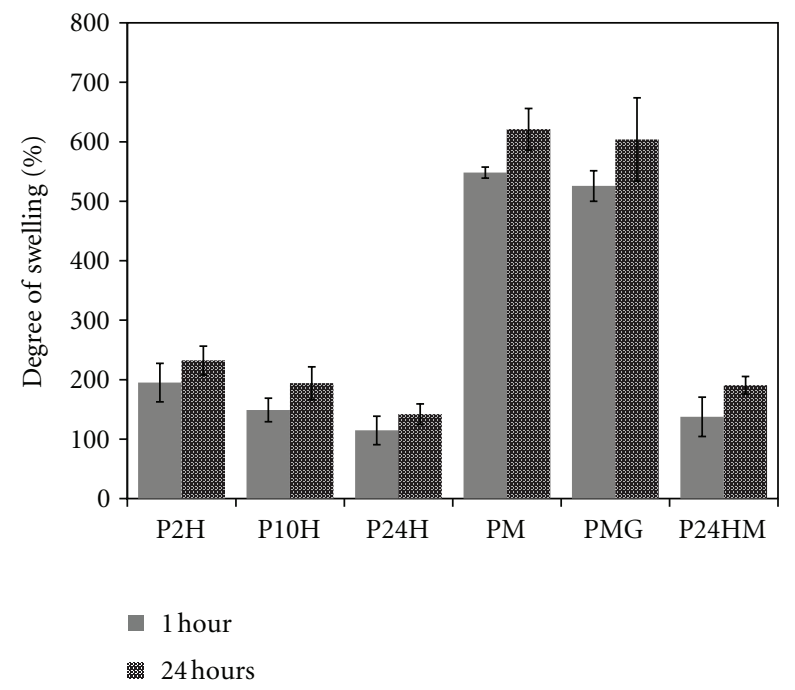

(a)

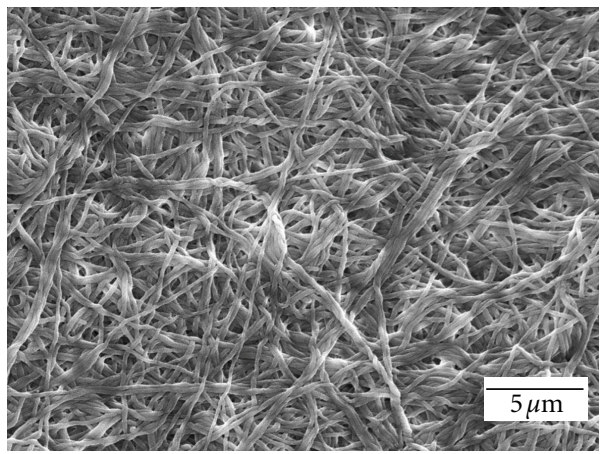

(b1)

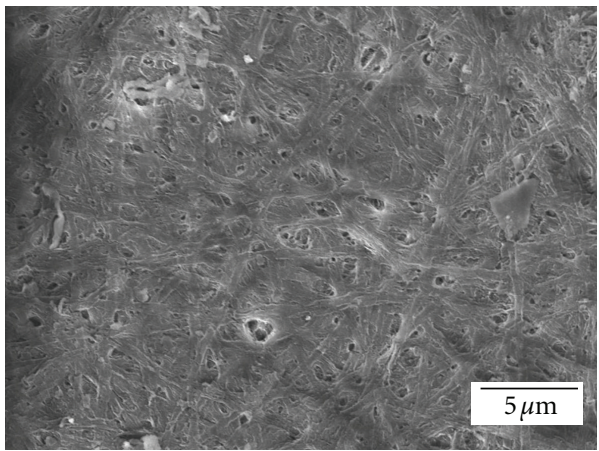

(b2)

Figure 4: Swelling rates of crosslinked PVPA/PVA membranes (a) and SEM micrographs of P24H (b1) and PM (b2) after 1-week PBS immersion.

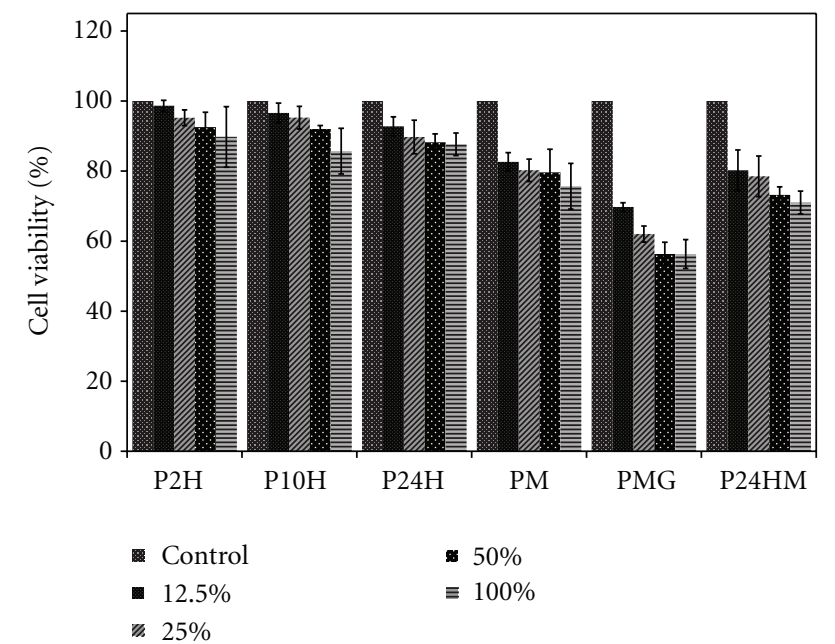

FIGURE 5: Determination of material cytotoxicity of heat-crosslinked membranes $(\mathrm{P} 2 \mathrm{H}, \mathrm{P} 10 \mathrm{H}, \mathrm{P} 24 \mathrm{H})$, methanol and methanol/glutaraldehyde-treated membranes (PM, PMG) and membrane crosslinked with combined methods (P24HM) using MTT assay.

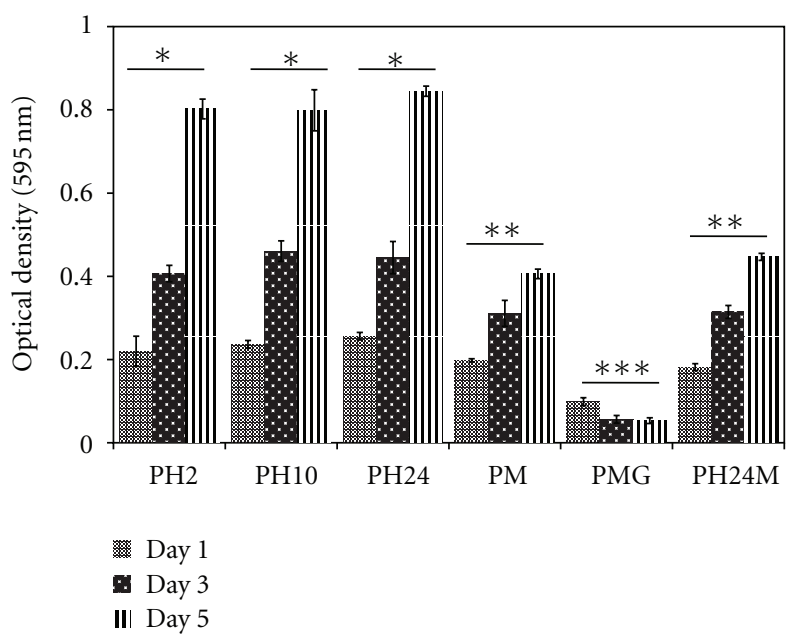

Figure 6: Cell proliferation of MG63 osteoblast-like cells on crosslinked PVPA/PVA membranes assessed by MTT assay. Heatcrosslinked membranes $(*)$ showed cell growth that are higher than membranes exposed to chemical crosslinking $\left(^{* *}\right)$ with PMG showing reduced growth during prolonged incubation time $\left(^{* * *}\right)(P>$ 0.05). 


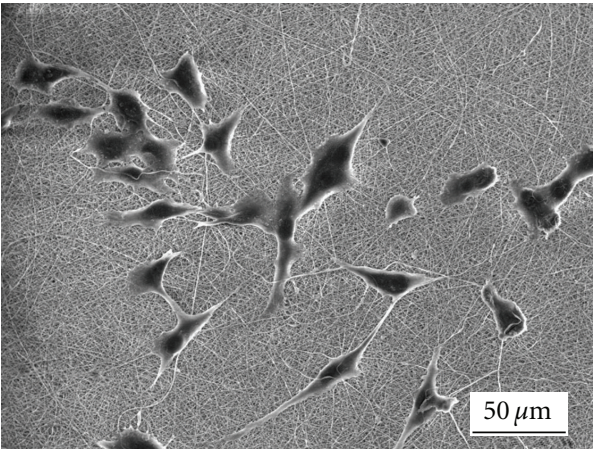

(a1)

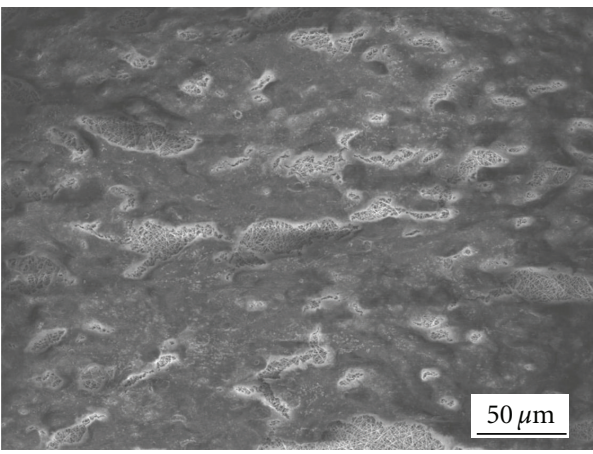

(a2)

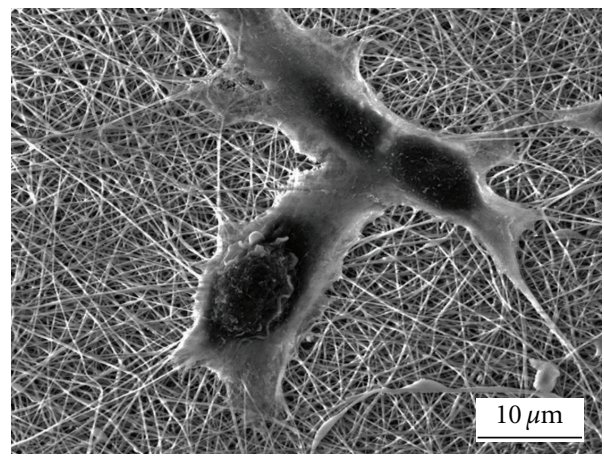

(b1)

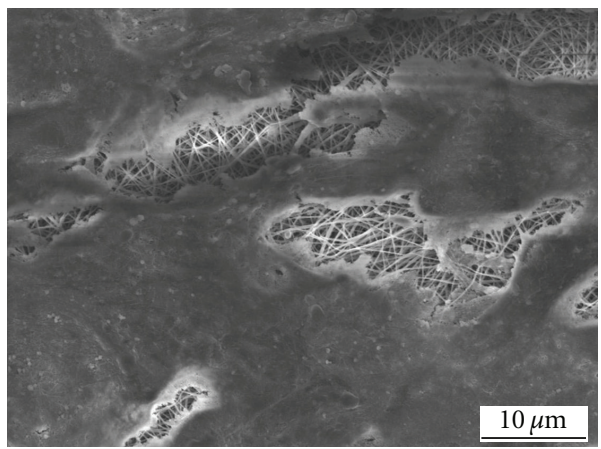

(b2)

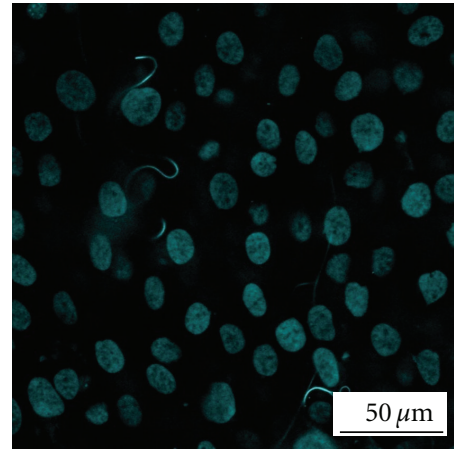

(c1)

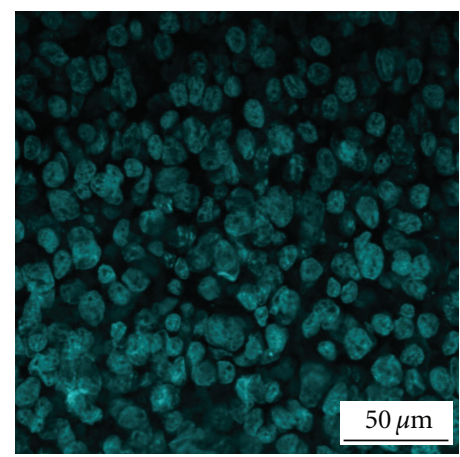

(c2)

Figure 7: SEM micrographs (a), magnified images (b), and confocal images (c) of MG63 osteoblast-like cells showing adherence on P24H after 1 day (1) and 5 days (2).

its ability to bind to natural bone hydroxyapatite because of the abundance of phosphonate groups, which shares similar structural formula with phosphates [9]. This is the first study where PVPA was used in the electrospinning method. PVPA is highly hydrophilic and quickly reacts with water. However, when it was combined with PVA, a highly miscible solution was produced due to its shared affinity for water.

PVA fibers are known to be stable at a temperature of $150^{\circ} \mathrm{C}[21]$ and copolymer PVPA membranes showed high thermal stability at temperatures up to $300^{\circ} \mathrm{C}$ [9]. Heat treatment, which exposes the PVPA/PVA membrane to a relatively high temperature of $150^{\circ} \mathrm{C}$, dehydrates the membrane since water starts to evaporate at $100^{\circ} \mathrm{C}$. The phosphonic acid in PVPA does not self-condense at $150^{\circ} \mathrm{C}$ [9]. The elevated temperature resulted in the formation of crystals that rendered the PVA insoluble in water [22].

The fabricated PVPA/PVA membranes contained uniform and consistent fibers that were randomly deposited on the collecting surface. PVPA is acidic, which may have contributed to its high conductivity [6] and facilitated the formation of uniform fibers at collection. The fabricated membrane quickly dissolved in water; thus physical and chemical treatments were performed in order to stabilize the membrane. Exposure to high temperature produced slight changes in the fiber microstructure since dehydrated fibers became flattened and compressed against each other (Figure 2(b)). Moreover, a notable discoloration were observed on the macroscopic membrane surface, which increased with intensity with exposure time (Figure 1(a)). The discoloration may be due to the acids of PVPA, which were deposited on the membrane surface when the water evaporated during heat treatment. Increased exposure time further released more water and, consequently, a greater amount of acidic residues was observed on the surface.

Chemical crosslinking allows the linking of one polymer bond to another in order to enhance material properties that will make it more suitable for a desired function. Previous studies have used glutaraldehyde and hydrochloric acid [23], UV light exposure [24, 25] hexamethyl diisocyanate [26], and maleic acid with heat treatment [27], which all produced more favorable material characteristics for PVA than its noncrosslinked counterparts. PVPA, on the other hand, had been crosslinked with different aldehydes to increase compressive strength [7]. Methanol crosslinks the hydroxyl groups of both PVA and PVPA. In a previous study by Yao et. al., it was shown that methanol dehydrates the PVA which replaced the polymer-water hydrogen with intermolecular polymer hydrogen bonding [28]. While this principle theoretically works for both polymers, significant changes were observed in both membrane microstructure and macrostructure. Immersion in methanol dehydrated the membrane which affected the membrane macrostructure, which contributed to membrane shrinkage upon drying (Figure 1). Membrane fibers are highly compressed against each other due to the high evaporative rate of methanol. Reimmersion in PBS allowed water molecules to enter the 
fiber network, which increased the high swelling rates and affected the swollen fiber morphology (Figure 4).

Addition of glutaraldehyde may produce a more stable membrane [29] since it theoretically crosslinks the free $-\mathrm{OH}$ groups of PVA and PVPA to the aldehyde group of glutaraldehyde more efficiently. While the stress values of PM and PMG were similar, the material strains of the two samples were statistically different. Immersion in methanol alone produced a more brittle membrane compared to when glutaraldehyde was present. Additional crosslinkers may have allowed more deformation without breaking. When compared with the heat-stabilized samples, the mechanical properties of methanol-crosslinked membranes were about fourfold higher. This may be due to more stable ionic bonding between the $-\mathrm{OH}$ groups of the PVPA and PVA with methanol and, subsequently, with the aldehyde groups of glutaraldehyde in PGM compared to heat stabilization of $\mathrm{P} 2 \mathrm{H}, \mathrm{P} 10 \mathrm{H}$, and $\mathrm{P} 24 \mathrm{H}$. The $\mathrm{P} 24 \mathrm{M}$ membrane had similar stress and strain values of the initially heated membranes with few discrepancies. After heat exposure, methanol may not have been able to bind with the polymers due to dehydration after treatment.

PVPA and PVA are both hydrophilic polymers and highly dissolvable in water. Treatment of the membranes prevented dissolution in water but allowed water permeation within the fiber structure as indicated by the increased weight and relative increase in diameter following PBS immersion (Figure 4). Both heat treatment and methanol immersion dehydrate the membrane fibers, which rendered them insoluble in water. When membranes were immersed in PBS, the samples were rehydrated but the rate of rehydration depended on the length of heat exposure and method used. At longer heating time, membranes exhibited reduced swelling, which may be due to a higher degree of membrane crystallization when exposure to heat occurred at higher temperature or longer exposure time [27].

Material biocompatibility is an important factor in the utilization of tissue engineering scaffolds. When investigating the material cytotoxicity of the crosslinked membranes, extract solutions were obtained and used as treatment for a commercial cell line in accordance with the ISO-10993-5 protocol. In this study, heat-stabilized membrane displayed relatively higher cell viability compared to the membranes exposed to methanol (PM, P24HM) and methanol/glutaraldehyde (PMG). Chemical residues on the membranes may have affected cell adhesion on the membrane surface, which could have reduced cell viability (Figure 5) and, subsequently, decreased the proliferation rate (Figure 6).

Material properties (except for the strain values and swelling), cytotoxicity, and cell proliferation rates were relatively similar for all heat-treated membranes. Thus, the heat-treated $\mathrm{P} 24 \mathrm{H}$ membrane was used to determine the cell adhesion behavior of MG63 osteoblast-like cells in consideration of its potential to maintain its integrity because of its reduced swelling rate. It was observed on that the 5th day of culture, the extracellular material almost completely covered the membrane surface, indicating the potential of this material as a tissue engineering scaffold.

\section{Conclusion}

A novel nanofiber membrane composed of PVPA and PVA was fabricated by electrospinning method. Resulting membrane necessitated treatment due to high dissolvability in water. Two methods of stabilization were compared. Physical treatment by heat was done by exposing membranes to $150^{\circ} \mathrm{C}$ at different lengths of time. Chemical crosslinking was done by methanol and methanol/glutaraldehyde immersion. A membrane sample was also prepared with combined treatment methods. Membrane morphology was observed to be affected as discoloration intensified with prolonged heating period and surface area reduction and fiber morphology was significantly changed after chemical exposure. Mechanical strength as well as swelling and degradation was affected by the type of treatment employed wherein these properties were more pronounced when membranes were chemically modified. Biocompatibility was seen to significantly reduce in chemically crosslinked membranes as it showed higher cytotoxicity rates and reduced proliferation.

\section{Acknowledgment}

This work was supported by Midcareer Researcher Program through NRF Grant funded by MEST (no. 2009-0092808), Republic of Korea.

\section{References}

[1] R. Vasita and D. S. Katti, "Nanofibers and their applications in tissue engineering," International Journal of Nanomedicine, vol. 1, no. 1, pp. 15-30, 2006.

[2] J. H. Jang, O. Castano, and H. W. Kim, "Electrospun materials as potential platforms for bone tissue engineering," Advanced Drug Delivery Reviews, vol. 61, no. 12, pp. 1065-1083, 2009.

[3] Q. P. Pham, U. Sharma, and A. G. Mikos, "Electrospinning of polymeric nanofibers for tissue engineering applications: a review," Tissue Engineering, vol. 12, no. 5, pp. 1197-1211, 2006.

[4] Z. M. Huang, Y. Z. Zhang, M. Kotaki, and S. Ramakrishna, "A review on polymer nanofibers by electrospinning and their applications in nanocomposites," Composites Science and Technology, vol. 63, no. 15, pp. 2223-2253, 2003.

[5] A. O. Akinmade, J. H. Braybrook, and J. W. Nicholson, "Novel dental cements formed between polyvinyl phosphonic acid), PVPA, and heat treated zinc oxide," Journal of Materials Science Letters, vol. 13, no. 2, pp. 91-92, 1994.

[6] B. Bingöl, W. H. Meyer, M. Wagner, and G. Wegner, "Synthesis, microstructure, and acidity of poly(vinylphosphonic acid)," Macromolecular Rapid Communications, vol. 27, no. 20, pp. 1719-1724, 2006.

[7] J. H. Braybrook and J. W. Nicholson, "Incorporation of crosslinking agents into poly(vinyl phosphonic acid) as a route to glass-polyalkenoate cements of improved compressive strength," Journal of Materials Chemistry, vol. 3, no. 4, pp. 361365, 1993.

[8] Y. E. Greish and P. W. Brown, "Preparation and characterization of calcium phosphate-poly(vinyl phosphonic acid) composites," Journal of Materials Science: Materials in Medicine, vol. 12, no. 5, pp. 407-411, 2001.

[9] L. MacArie and G. Ilia, "Poly(vinylphosphonic acid) and its derivatives," Progress in Polymer Science, vol. 35, no. 8, pp. 1078-1092, 2010. 
[10] C. Shao, X. Yang, H. Guan, Y. Liu, and J. Gong, "Electrospun nanofibers of $\mathrm{NiO} / \mathrm{ZnO}$ composite," Inorganic Chemistry Communications, vol. 7, no. 5, pp. 625-627, 2004.

[11] H. Liao, R. Qi, M. Shen et al., "Improved cellular response on multiwalled carbon nanotube-incorporated electrospun polyvinyl alcohol/chitosan nanofibrous scaffolds," Colloids and Surfaces B, vol. 84, no. 2, pp. 528-535, 2011.

[12] E. Yang, X. Qin, and S. Wang, "Electrospun crosslinked polyvinyl alcohol membrane," Materials Letters, vol. 62, no. 20, pp. 3555-3557, 2008.

[13] D. Yang, Y. Li, and J. Nie, "Preparation of gelatin/PVA nanofibers and their potential application in controlled release of drugs," Carbohydrate Polymers, vol. 69, no. 3, pp. 538-543, 2007.

[14] E. R. Kenawy, F. I. Abdel-Hay, M. H. El-Newehy, and G. E. Wnek, "Controlled release of ketoprofen from electrospun poly(vinyl alcohol) nanofibers," Materials Science and Engineering A, vol. 459, no. 1-2, pp. 390-396, 2007.

[15] K. T. Givens, S. Kitada, A. K. Chen, J. Rothschiller, and D. A. Lee, "Proliferation of human ocular fibroblasts: an assessment of in vitro colorimetric assays," Investigative Ophthalmology and Visual Science, vol. 31, no. 9, pp. 1856-1862, 1990.

[16] S. Jiang, S. Liu, and W. Feng, "PVA hydrogel properties for biomedical application," Journal of the Mechanical Behavior of Biomedical Materials, vol. 4, no. 7, pp. 1228-1233, 2011.

[17] N. T. B. Linh, Y. K. Min, H. Y. Song, and B. T. Lee, "Fabrication of polyvinyl alcohol/gelatin nanofiber composites and evaluation of their material properties," Journal of Biomedical Materials Research Part B, vol. 95, no. 1, pp. 184-191, 2010.

[18] J. M. Yang, W. Y. Su, T. L. Leu, and M. C. Yang, "Evaluation of chitosan/PVA blended hydrogel membranes," Journal of Membrane Science, vol. 236, no. 1-2, pp. 39-51, 2004.

[19] F. R. Lamastra, A. Bianco, A. Meriggi, G. Montesperelli, F. Nanni, and G. Gusmano, "Nanohybrid PVA/Z $\mathrm{ZO}_{2}$ and $\mathrm{PVA} / \mathrm{Al}_{2} \mathrm{O}_{3}$ electrospun mats," Chemical Engineering Journal, vol. 145, no. 1, pp. 169-175, 2008.

[20] N. A. M. Zain, M. S. Suhaimi, and A. Idris, "Development and modification of PVA-alginate as a suitable immobilization matrix," Process Biochemistry, vol. 46, no. 11, pp. 2122-2129, 2011.

[21] L. A. Vol'f, Y. K. Kirilenko, Z. A. Urban, E. Y. Danilova, O. V. Bukalova, and G. I. Kudryavtsev, "Imparting thermal stability to fibres based on polyvinyl alcohol," Fibre Chemistry, vol. 1, no. 3, pp. 258-261, 1970.

[22] P. R. Byron and R. N. Dalby, "Effects of heat treatment on the permeability of polyvinyl alcohol films to a hydrophilic solute," Journal of Pharmaceutical Sciences, vol. 76, no. 1, pp. 65-67, 1987.

[23] C. Tang, C. D. Saquing, J. R. Harding, and S. A. Khan, "In situ cross-linking of electrospun poly(vinyl alcohol) nanofibers," Macromolecules, vol. 43, no. 2, pp. 630-637, 2010.

[24] A. Gupta, C. D. Saquing, M. Afshari, A. E. Tonelli, S. A. Khan, and R. Kotek, "Porous nylon-6 fibers via a novel salt-induced electrospinning method," Macromolecules, vol. 42, no. 3, pp. 709-715, 2009.

[25] S. H. Kim, S. H. Kim, S. Nair, and E. Moore, "Reactive electrospinning of cross-linked poly(2-hydroxyethyl methacrylate) nanofibers and elastic properties of individual hydrogel nanofibers in aqueous solutions," Macromolecules, vol. 38, no. 9, pp. 3719-3723, 2005.

[26] M. Krumova, D. López, R. Benavente, C. Mijangos, and J. M. Pereña, "Effect of crosslinking on the mechanical and thermal properties of poly(vinyl alcohol)," Polymer, vol. 41, no. 26, pp. 9265-9272, 2000.
[27] J. M. Gohil, A. Bhattacharya, and P. Ray, "Studies on the crosslinking of poly (vinyl alcohol)," Journal of Polymer Research, vol. 13, no. 2, pp. 161-169, 2006.

[28] L. Yao, T. W. Haas, A. Guiseppi-Elie, G. L. Bowlin, D. G. Simpson, and G. E. Wnek, "Electrospinning and stabilization of fully hydrolyzed poly(vinyl alcohol) fibers," Chemistry of Materials, vol. 15, no. 9, pp. 1860-1864, 2003.

[29] V. S. Praptowidodo, "Influence of swelling on water transport through PVA-based membrane," Journal of Molecular Structure, vol. 739, no. 1-3, pp. 207-212, 2005. 

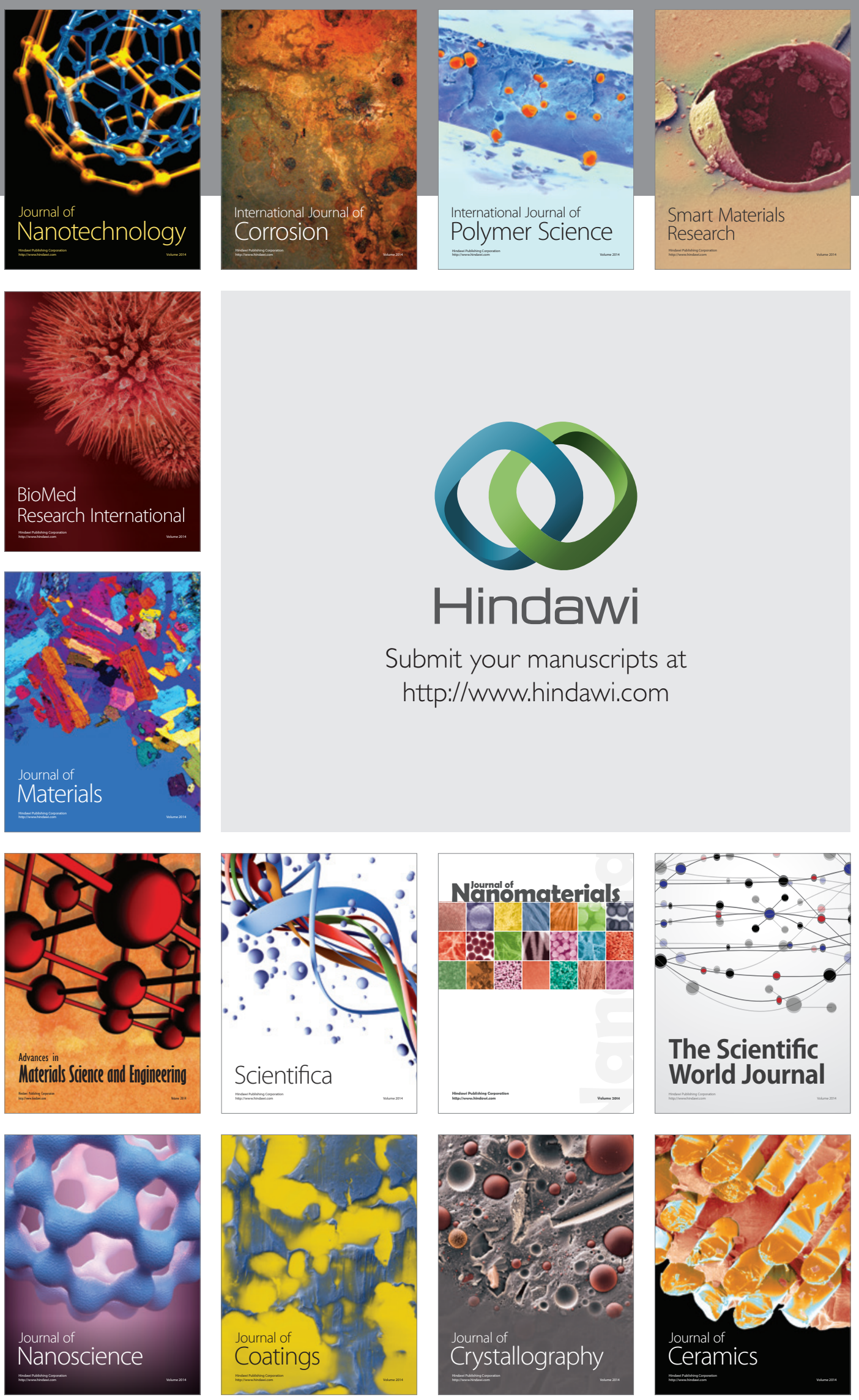

The Scientific World Journal

Submit your manuscripts at

http://www.hindawi.com

\section{World Journal}

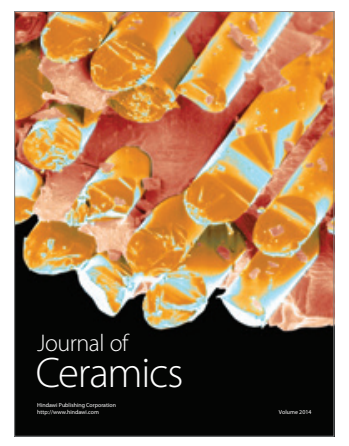

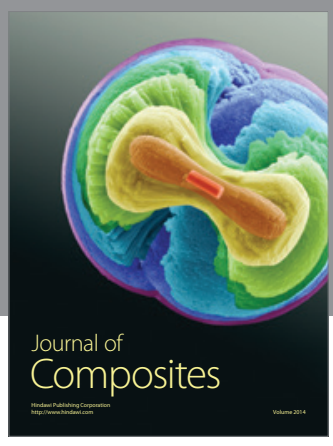
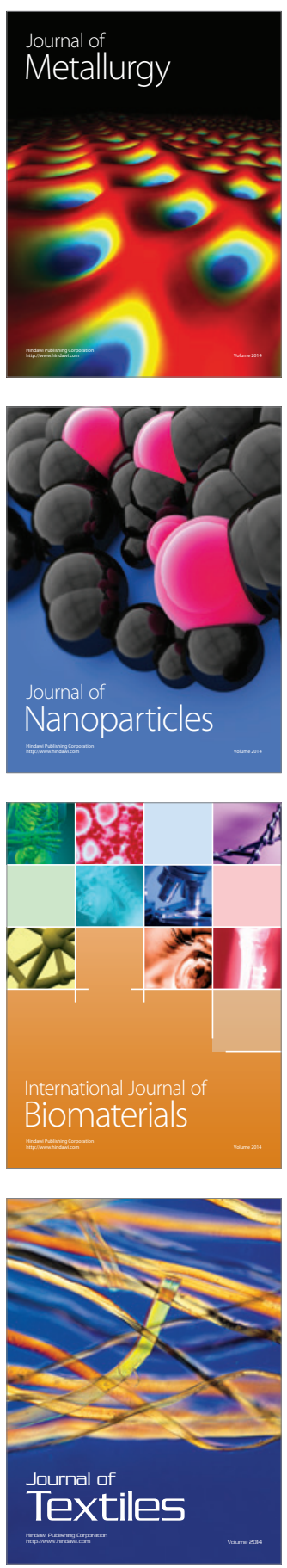